\title{
RYBAKOV'S THEOREM IN FRÉCHET SPACES AND COMPLETENESS OF $L^{1}$-SPACES
}

\author{
W. J. RICKER \\ (Received 28 April 1997; revised 10 October 1997)
}

Communicated by P. G. Dodds

\begin{abstract}
We provide a simple and direct proof of the completeness of the $L^{1}$-space of any vector measure taking its values in the class of Fréchet spaces which do not contain a copy of the sequence space $\omega$.
\end{abstract}

1991 Mathematics subject classification (Amer: Math. Soc.): primary $28 \mathrm{~B} 05$.

Bartle, Dunford and Schwartz [1] (respectively Kluvánek and Knowles [6], and Lewis [7]) developed integration theories in Banach (respectively locally convex) spaces in order to integrate scalar functions with respect to a vector measure. Kluvánek and Knowles in [6] and, more recently Curbera in [2,3] and Okada in [8], among others, have studied topological and order properties of the $L^{1}$-space of an $X$-valued vector measure $m$, with $X$ a vector space over $\mathbb{R}$. From the point of view of analysis it is most important to be able to decide when $L^{1}(m)$ is complete for its natural topology of uniform convergence of indefinite integrals. This question is intimately related to the theory of closed vector measures and forms a major part of the monograph [6]. Under mild completeness assumptions on the real space $X$ it is shown that $L^{1}(m)$ is complete if and only if $m$ is a closed measure. The method of proof is based on a careful and detailed analysis of various topologies induced on $L^{1}(m)$ by certain families of scalar measures and the restriction of these topologies to certain subsets of $L^{1}(m)$; see [6]. Nevertheless, as attractive and general as these completeness results of $L^{1}(m)$ are, it certainly cannot be claimed that the proofs are particularly transparent.

The aim of this note is to give a short, direct and transparent proof of the com-

This research was carried out while the author was a visitor in the Mathematisches Institut of the Johannes Kepler Universität Linz. The support of the Functional Analysis group from there is gratefully acknowledged.

(C) 1998 Australian Mathematical Society 0263-6115/98 \$A2.00+0.00 
pleteness of $L^{1}(m)$ for any vector measure $m$ taking its values in the class of Fréchet spaces which do not contain an isomorphic copy of the sequence space $\omega=\mathbb{C}^{\mathbb{N}}$. This is possible due to a recent result of Fernández and Naranjo [4] characterizing such (real) Fréchet spaces as precisely those in which Rybakov's theorem holds. This class of spaces contains all Banach spaces and so, in particular, we provide a simple and transparent proof of the completeness of the $L^{1}$-space for Banach space-valued measures. As far as the author is aware there does not seem to be such a 'simple' proof explicitly available in the literature even in this special case when $m$ is Banach space-valued (other than the general results in [6]).

In what follows we denote by $X$ a Fréchet space over $\mathbb{C}$ and by $\left\{q^{(r)}\right\}_{r=1}^{\infty}$ an increasing sequence of seminorms defining the topology of $X$. Consider a countably additive measure $m: \Sigma \rightarrow X$ defined on a $\sigma$-algebra $\Sigma$ of subsets of some set $\Omega$. For each $x^{\prime} \in X^{\prime}$ (the continuous dual space of $X$ ), let $\left\langle m, x^{\prime}\right\rangle: \Sigma \rightarrow \mathbb{C}$ denote the complex measure $E \mapsto\left\langle m(E), x^{\prime}\right\rangle$ for $E \in \Sigma$. The $q^{(r)}$-semivariation of $m$ is defined to be the function $q^{(r)}(m): \Sigma \rightarrow[0, \infty)$ given by

$$
q^{(r)}(m)(E)=\sup \left\{\left|\left\langle m, x^{\prime}\right\rangle\right|(E): x^{\prime} \in U_{r}^{\circ}\right\}, \quad E \in \Sigma,
$$

for each $r \in \mathbb{N}$, where $U_{r}^{\circ}$ is the polar of $\left\{x \in X: q^{(r)}(x) \leq 1\right\}$ and $\left|\left\langle m, x^{\prime}\right\rangle\right|$ is the total variation measure of $\left\langle m, x^{\prime}\right\rangle$. Then

$$
\begin{aligned}
\sup \left\{q^{(r)}(m(F)): F \in \Sigma, F \subseteq E\right\} & \leq q^{(r)}(m)(E) \\
& \leq 4 \sup \left\{q^{(r)}(m(F)): F \in \Sigma, F \subseteq E\right\}
\end{aligned}
$$

for each $E \in \Sigma$ and $r \in \mathbb{N}$; see [7].

Let $m: \Sigma \rightarrow X$ be a vector measure and $\mu: \Sigma \rightarrow[0, \infty)$ be a finite measure. Then we say that $m$ is absolutely continuous with respect to $\mu$, denoted by $m \ll \mu$, if and only if $m(E)=0$ whenever $\mu(E)=0$. This is equivalent to $q^{(r)}(m)(E)=0$, for all $r \in \mathbb{N}$, if and only if $\mu(E)=0$ which, in turn, is equivalent to $m(E) \rightarrow 0$ in $X$ if and only if $\mu(E) \rightarrow 0, E \in \Sigma$. A Fréchet space $X$ is said to have Rybakov's property [4] if for every $X$-valued vector measure $m$ there is $x^{\prime} \in X^{\prime}$ such that $m \ll\left|\left\langle m, x^{\prime}\right\rangle\right|$. The classical theorem of Rybakov [9] states that Banach spaces have the Rybakov property. The following result is an extension of Rybakov's theorem to a class of Fréchet spaces; it was established (amongst other interesting results) in [4] for all real Fréchet spaces not containing a copy of $\omega_{\mathbb{R}}=\mathbb{R}^{\mathbb{N}}$. The argument is based on the existence of exposed points in the range of $m$. The usual 'complexification argument' can be applied to extend this result to complex Fréchet spaces. However, we prefer here to give an alternate proof which applies directly in both real and complex spaces.

PROPOSITION 1. A Fréchet space $X$ has Rybakov's property if and only if it does not contain an isomorphic copy of $\omega$. 
PROOF. If $X$ contains a copy of $\omega$, then it contains a complemented copy of $\omega$ [5; Theorem 7.2.7]. It is then straightforward to exhibit an $X$-valued measure for which no Rybakov functional $x^{\prime} \in X^{\prime}$ exists; see Examples 1.1 and 1.2 in [4].

Conversely, suppose that $X$ does not contain a copy of $\omega$. Then $X$ admits a continuous norm $\|\cdot\|: X \rightarrow[0, \infty)$, [5; Theorem 7.2.7]. Let $m: \Sigma \rightarrow X$ be any vector measure. If $X_{\|\cdot\|}$ denotes $X$ equipped with the norm topology induced by $\|\cdot\|$, then $m: \Sigma \rightarrow X_{\|\cdot\| \|}$ is also $\sigma$-additive. Let $\tilde{X}_{\|\cdot\| \|}$ be the (Banach space) completion of $X_{\|\cdot \cdot\|}$ in which case $m$ is still $\sigma$-additive when considered as being $\tilde{X}_{\|\cdot\|}$-valued. By the classical Rybakov theorem for Banach spaces there is $\xi \in\left(\tilde{X}_{\|\cdot\| \cdot \|}\right)^{\prime}$ such that $m \ll|\langle m, \xi\rangle|$, that is, $m(E)=0$ in $\tilde{X}_{\|\cdot\|}$ whenever $|\langle m, \xi\rangle|(E)=0$. Of course, the restriction $\xi_{0}$ of $\xi$ to $X$ is an element of $\left(X_{\|\cdot\|}\right)^{\prime}$. Since

$$
\left|\left\langle x, \xi_{0}\right\rangle\right| \leq C_{1}\|x\| \leq C_{2} q^{(r)}(x), \quad x \in X,
$$

for some $r \in \mathbb{N}$ and constants $C_{j}>0$ it is clear that $\xi_{0} \in X^{\prime}$. But, $m(E)=0$ in $X_{\|\cdot\|} \subseteq \tilde{X}_{\|\cdot\|}$ if and only if $m(E)=0$ in $X$ (as $\|\cdot\|$ is a norm in $X$ ) and so also $m \ll\left|\left\langle m, \xi_{0}\right\rangle\right|$ when $m$ is considered as $X$-valued. Hence, $\xi_{0}$ is Rybakov functional for $m$.

The following technical result [7; Lemma 2.3] will be needed later; it is a consequence of the Vitali-Hahn-Saks theorem.

LeMma 1. Let $v: \Sigma \rightarrow \mathbb{C}$ be a complex measure on a $\sigma$-algebra $\Sigma$. Let $f_{n}$ : $\Omega \rightarrow \mathbb{C}, n=1,2, \ldots$, be a sequence of $\nu$-integrable functions and $f: \Omega \rightarrow \mathbb{C}$ be a function such that

(i) $\lim _{n \rightarrow \infty} f_{n}(w)=f(w)$, for each $w \in \Omega$, and

(ii) $\left\{\int_{E} f_{n} d \nu\right\}_{n=1}^{\infty}$ is Cauchy in $\mathbb{C}$, for each $E \in \Sigma$.

Then $f \in L^{1}(v)$ and $f_{n} \rightarrow f$ in $L^{1}(v)$.

DEFINITION 1. Let $m: \Sigma \rightarrow X$ be a vector measure. A $\Sigma$-measurable function $f: \Omega \rightarrow \mathbb{C}$ is called $m$-integrable if

(i) $f \in L^{1}\left(\left\langle m, x^{\prime}\right\rangle\right)$, for each $x^{\prime} \in X^{\prime}$, and

(ii) for each $E \in \Sigma$ there exists an (necessarily unique) element of $X$, denoted by $\int_{E} f d m$, which satisfies

$$
\left\langle\int_{E} f d m, x^{\prime}\right\rangle=\int_{E} f d\left\langle m, x^{\prime}\right\rangle, \quad x^{\prime} \in X^{\prime} .
$$

The Orlicz-Pettis theorem ensures that if $f$ is $m$-integrable, then the set function $m_{f}: \Sigma \rightarrow X$ defined by $m_{f}(E)=\int_{E} f d m$, for each $E \in \Sigma$, is again a vector measure; it is called the indefinite integral of $f$ with respect to $m$. If $m_{f}$ happens to 
be the zero vector measure, then $f$ is said to be an $m$-null function. In particular, if $f=\chi_{E}$ is an $m$-null function, then we say $E$ is an $m$-null set. Two $m$-integrable functions $f$ and $g$ are called $m$-equivalent if $|f-g|$ is $m$-null. In the usual way $L^{\prime}(m)$ then denotes the space of all equivalence classes (modulo $m$-equivalence) of $m$-integrable functions. It becomes a (metrizable) locally convex Hausdorff space equipped with the sequence of seminorms

$$
\|f\|_{r}=q^{(r)}\left(m_{f}\right)(\Omega), \quad f \in L^{1}(m),
$$

for each $r \in \mathbb{N}$. As noted above the following result is known for real Fréchet spaces; see IV.4 Theorem 1 and IV.7 Theorem 1 in [6].

PROPOSITION 2. Let $X$ be a Fréchet space and $m: \Sigma \rightarrow X$ be a vector measure. Then $L^{1}(m)$ is complete and hence, in particular, is again a Fréchet space.

As mentioned before our aim is to give a simple and direct proof of Proposition 2 in the case when the complex Fréchet space $X$ does not contain a copy of $\omega$.

So, suppose that this is the case. By Proposition 1 there is $x^{\prime} \in X^{\prime}$ with $m \ll$ $\left|\left\langle m, x^{\prime}\right\rangle\right|$. Let $\left\{f_{n}\right\}_{n=1}^{\infty}$ be a Cauchy sequence in $L^{\prime}(m)$. Since $x^{\prime}: X \rightarrow \mathbb{C}$ is continuous there is a constant $\alpha>0$ and $r(0) \in \mathbb{N}$ such that $\left|\left\langle x, x^{\prime}\right\rangle\right| \leq \alpha q^{(r(0))}(x)$, for each $x \in X$. By considering $\alpha^{-1} x^{\prime}$ and noting that $m \ll\left|\left\langle m, \alpha^{-1} x^{\prime}\right\rangle\right|$ we may suppose that $\alpha=1$. Hence, $\left|\left\langle x, x^{\prime}\right\rangle\right| \leq q^{(r(0))}(x)$ for $x \in X$ and so $x^{\prime} \in U_{r(0)}^{\circ}$. Accordingly, by (1) and (3) we have that

$$
\int_{\Omega}\left|f_{k}-f_{n}\right| d\left|\left\langle m, x^{\prime}\right\rangle\right| \leq\left\|f_{k}-f_{n}\right\|_{r(0)}, \quad k, n \in \mathbb{N},
$$

which shows that $\left\{f_{n}\right\}_{n=1}^{\infty}$ is Cauchy in $L^{1}\left(\left\langle m, x^{\prime}\right\rangle\right)$. Hence, there is $f \in L^{1}\left(\left\langle m, x^{\prime}\right\rangle\right)$ such that $f_{n} \rightarrow f$ in $L^{\prime}\left(\left\langle m, x^{\prime}\right\rangle\right)$. Then there is a subsequence $\left\{f_{n(k)}\right\}_{k=1}^{\infty}$ with $f_{n(k)} \rightarrow f$ pointwise $\left|\left\langle m, x^{\prime}\right\rangle\right|-$ a.e. on $\Omega$, and hence also $m$ - a.e. on $\Omega$. That is, there is an $m$-null set $A \in \Sigma$ such that $f_{n(k)}(w) \rightarrow f(w)$ for all $w \in \Omega \backslash A$. If necessary redefine $f$ and each $f_{n(k)}, k \in \mathbb{N}$, to be zero on this $m$-null set so that $f_{n(k)} \rightarrow f$ pointwise everywhere on $\Omega$.

Fix $y^{\prime} \in X^{\prime}$. Then there is $\beta>0$ and $j \in \mathbb{N}$ such that $\left|\left\langle x, y^{\prime}\right\rangle\right| \leq \beta q^{(j)}(x)$ for all $x \in X$. In particular, $\beta^{-1} y^{\prime} \in U_{j}^{\circ}$. So, for any fixed $E \in \Sigma$ we have

$$
\begin{aligned}
\left|\int_{E} f_{n(k)} d\left\langle m, y^{\prime}\right\rangle-\int_{E} f_{n(l)} d\left\langle m, y^{\prime}\right\rangle\right| & \leq \beta \int_{E}\left|f_{n(k)}-f_{n(l)}\right| d\left|\left\langle m, \beta^{-1} y^{\prime}\right\rangle\right| \\
& \leq \beta\left\|f_{n(k)}-f_{n(l)}\right\|_{j},
\end{aligned}
$$

for all $k, l \in \mathbb{N}$, which shows that $\left\{\int_{E} f_{n(k)} d\left\langle m, y^{\prime}\right\rangle\right\}_{k=1}^{\infty}$ is Cauchy in $\mathbb{C}$. Then Lemma 1 implies that $f \in L^{1}\left(\left\langle m, y^{\prime}\right\rangle\right)$ and

$$
\lim _{k \rightarrow \infty} \int_{\Omega}\left|f-f_{n(k)}\right| d\left|\left\langle m, y^{\prime}\right\rangle\right|=0
$$


Fix $r \in \mathbb{N}$. Then for each fixed $E \in \Sigma$ we have

$$
\begin{aligned}
q^{(r)}\left(\int_{E} f_{n(k)} d m-\int_{E} f_{n(l)} d m\right) & =\sup \left\{\left|\left\langle\int_{E}\left(f_{n(k)}-f_{n(l)}\right) d m, z^{\prime}\right\rangle\right|: z^{\prime} \in U_{r}^{\circ}\right\} \\
& \leq \sup \left\{\int_{\Omega}\left|f_{n(k)}-f_{n(l)}\right| d\left|\left\langle m, z^{\prime}\right\rangle\right|: z^{\prime} \in U_{r}^{\circ}\right\} \\
& =\left\|f_{n(k)}-f_{n(l)}\right\|_{r},
\end{aligned}
$$

for all $k, l \in \mathbb{N}$. Since $\left\{f_{n(k)}\right\}_{k=1}^{\infty}$ is Cauchy in $L^{1}(m)$ it follows that $\left\{\int_{E} f_{n(k)} d m\right\}_{k=1}^{\infty}$ is Cauchy in $X$ and so there exists $x_{E} \in X$ such that $\int_{E} f_{n(k)} d m \rightarrow x_{E}$ (in $\left.X\right)$ as $k \rightarrow \infty$. Define $\int_{E} f d m:=x_{E}$. Then

$$
\left\langle\int_{E} f d m, y^{\prime}\right\rangle=\lim _{k \rightarrow \infty}\left\langle\int_{E} f_{n(k)} d m, y^{\prime}\right\rangle=\lim _{k \rightarrow \infty} \int_{E} f_{n(k)} d\left\langle m, y^{\prime}\right\rangle=\int_{E} f d\left\langle m, y^{\prime}\right\rangle,
$$

where the last equality follows from (4). Since $E \in \Sigma$ and $y^{\prime} \in X^{\prime}$ are arbitrary we see that the requirements of Definition 1 are satisfied, that is $f \in L^{1}(m)$.

Since $\left\{f_{n}\right\}_{n=1}^{\infty}$ is Cauchy in $L^{1}(m)$ to show that $f_{n} \rightarrow f$ in $L^{1}(m)$ it suffices to show that the subsequence $\left\{f_{n(k)}\right\}_{k=1}^{\infty}$ converges to $f$ in $L^{1}(m)$. So, fix $\epsilon>0$. Given $r \in \mathbb{N}$ there exists $K \in \mathbb{N}$ such that

$$
\left\|f_{n(k)}-f_{n(l)}\right\|_{r} \leq \epsilon / 4, \quad k, l \geq K .
$$

Fix $E \in \Sigma$. If $k \geq K$ then the left-hand inequality in (2) with $m_{\left(f_{n(t)}-f_{m(t)}\right)}$ in place of $m$, together with (5) implies that

$$
\text { (6) } q^{(r)}\left(\int_{E} f d m-\int_{E} f_{n(k)} d m\right)=\lim _{l \rightarrow \infty} q^{(r)}\left(\int_{E} f_{n(l)} d m-\int_{E} f_{n(k)} d m\right) \leq \epsilon / 4 \text {. }
$$

But then the right-hand inequality in (2), now with $m_{\left(f-f_{n(k)}\right)}$ in place of $m$, together with (6) implies that $\left\|f-f_{n(k)}\right\|_{r} \leq \epsilon$, for all $k \geq K$. Accordingly, $\left\|f-f_{n(k)}\right\|_{r} \rightarrow 0$ as $k \rightarrow \infty$. Since $r \in \mathbb{N}$ is arbitrary we see that $f_{n(k)} \rightarrow f$ in $L^{1}(m)$ and the proof is complete.

\section{References}

[1] R. G. Bartle, N. Dunford and J. T. Schwartz, 'Weak compactness and vector measures', Canad. J. Math. 7 (1955), 289-305.

[2] G. P. Curbera, 'Operators into $L^{\prime}$ of a vector measure and applications to Banach lattices', Math. Ann. 292 (1992), 317-330.

[3] - 'When $L^{\prime}$ of a vector measure is an $A L$-space', Pacific J. Math. 162 (1994), 287-303.

[4] A. Fernández and F. Naranjo, 'Rybakov's theorem for vector measures in Fréchet spaces', Indag. Math. (New Series) 8 (1997), 33-42. 
[5] H. Jarchow, Locally convex spaces (Teubner, Stuttgart, 1981).

[6] I. Kluvánek and G. Knowles, Vector measures and control systems (North Holland, Amsterdam, 1975).

[7] D. R. Lewis, 'Integration with respect to vector measures', Pacific J. Math. 33 (1970), 157-165.

[8] S. Okada, 'The dual space of $L^{1}(\mu)$ for a vector measure $\mu$ ', J. Math. Anal. Appl. 177 (1993), 583-599.

[9] V. I. Rybakov, 'Theorem of Bartle, Dunford and Schwartz on vector-valued measures', Mat. Zametki 7 (1970), 247-254. English translation: Math. Notes 7, 147-151.

School of Mathematics

University of New South Wales

Sydney, NSW 2052

Australia 